\title{
TESTE DE CONDUTIVIDADE ELÉTRICA PARA SEMENTE DE PIMENTA ${ }^{1}$
}

\author{
DEBORAH DE SOUZA VIDIGAL ${ }^{2}$, JÚLIEN DA SILVA LIMA², MARIA CARMEN BHERING ${ }^{3}$, DENISE CUNHA F. SANTOS DIAS ${ }^{4}$
}

\begin{abstract}
RESUMO - O presente trabalho teve como objetivo verificar o efeito do número de sementes, do volume de água e do período de embebição no teste de condutividade elétrica para avaliar o vigor de cinco lotes de semente de pimenta e relacionar esses resultados com os testes de germinação, primeira contagem, envelhecimento acelerado e comprimento de plântula. O teste de condutividade elétrica foi realizado com 25 e 50 sementes embebidas em $25 \mathrm{~mL}$ e $50 \mathrm{~mL}$ de água destilada, a $25^{\circ} \mathrm{C}$, por $1,2,3$, $4,5,6,7,8,12,16,24$ e 48 horas. O teste de condutividade elétrica foi eficiente para a avaliação do vigor de sementes de pimenta, porém a utilização de 25 sementes não permitiu diferenciar os lotes. Para se obter melhor estratificação dos lotes em níveis de vigor, recomenda-se utilizar amostras de 50 sementes, embebidas em $25 \mathrm{~mL}$ de água destilada e avaliar a condutividade elétrica após uma hora de embebição.
\end{abstract}

Termos para indexação: Capsicum annuum, qualidade, vigor.

ELECTRICAL CONDUCTIVITY TEST FOR PEPPER SEEDS

\begin{abstract}
This study was carried out with the objective of verifying the effect of pepper seeds number, water volume and imbibition period on the electrical conductivity test results and correlating these data with germination, first count, accelerated aging and seedling length tests results. The electrical conductivity test was conducted with 25 and 50 seeds per replication, imbibed in 25 and $50 \mathrm{~mL}$ of water at $25^{\circ} \mathrm{C}$ for $1,2,3,4,5,6,7,8,12,16,24$, and 48 hours. The electrical conductivity test was efficient to evaluate pepper seeds vigor, but 25 showed to be an inadequate number of seeds when the objective was to identify seed lot vigor level. Better results were obtained when samples of 50 seeds, soaked for 1 hour in $25 \mathrm{~mL}$ of water were used.
\end{abstract}

Index terms: Capsicum annuum, quality, vigor.

\section{INTRODUÇÃO}

A utilização de sementes de alta qualidade é o fator predominante para o sucesso de qualquer cultura; por isso, vários testes de vigor são indispensáveis dos laboratórios de análises de sementes.

Nos programas de controle de qualidade interno das empresas produtoras de sementes a rapidez na obtenção

${ }^{1}$ Submetido em 06/09/2007. Aceito para publicação em 09/01/2008.

${ }^{2}$ Estudante de Pós-Graduação, Depto. de Fitotecnia, Universidade Federal de Viçosa, 36571-000 - Viçosa - MG. E-mail: dsvidigal@gmail.com; julienslima@gmail.com dos resultados é um fator de extrema importância (Dias \& Marcos Filho, 1996).

O teste de condutividade elétrica é rápido, prático e fácil de ser conduzido para determinação do vigor de sementes, não necessitando de muitos equipamentos e treinamento pessoal (Vieira \& Krzyzanowski, 1999). Diante disto, várias pesquisas vêm sendo desenvolvidas no sentido de reduzir o período de embebição para a avaliação da condutividade
${ }^{3}$ Pesquisadora, M.S., Depto. de Fitotecnia, Universidade Federal de Viçosa, 36571-000 - Viçosa - MG. E-mail: mbhering@ufv.br

${ }^{4}$ Prof. Associado, Depto. de Fitotecnia, , Universidade Federal de Viçosa, 36571-000 - Viçosa - MG. E-mail: dcdias@ufv.br 
elétrica em diferentes espécies, tais como amendoim (Vanzolini e Nakagawa, 1998), feijão de vagem (Dias et al., 1998), quiabo (Dias et al. 1998), tomate (Rodo et al., 1998), pimentão (Roveri-José et al., 2001 e Oliveira e Novembre, 2005), abobrinha (Dutra e Vieira, 2006) e cebola (Dias et al., 2006).

O valor da condutividade elétrica medida na solução de embebição de sementes é função da quantidade de íons lixiviados, estando diretamente relacionado com a integridade das membranas celulares. Desta forma, membranas mal estruturadas e células danificadas estão, geralmente, associadas com o processo de deterioração da semente, ou seja, com sementes de baixo vigor (Vieira e Krzyzanowski, 1999; AOSA, 2002).

Alguns fatores podem afetar os resultados do teste de condutividade elétrica, tais como: qualidade da água, temperatura e duração do período de embebição, grau de umidade da semente, número de sementes testadas e genótipo (Dias e Marcos Filho, 1996; Vieira e Krzyzanowski, 1999; Gaspar e Nakagawa, 2002).

Para a condução do teste de condutividade elétrica, a AOSA recomenda quatro repetições de 25 sementes; no entanto, Loeffler et al. (1998) propõem a utilização de quatro repetições de 50 sementes como forma de reduzir o coeficiente de variação, obtendo-se maior uniformidade dos resultados entre repetições. Alguns trabalhos visando adequar o teste à determinada espécie, avaliaram diferentes números de sementes. Gaspar e Nakagawa (2002), trabalhando com sementes de milheto, concluíram que a melhor combinação para a realização do teste de condutividade elétrica foi de 100 sementes e $100 \mathrm{~mL}$ de água destilada a $25^{\circ} \mathrm{C}$ por 24 horas, diferenciando os lotes quanto à qualidade fisiológica, além de apresentar menor coeficiente de variação. Rodo et al. (1998) observaram em sementes de tomate que, o teste de condutividade elétrica foi mais eficiente para o cultivar IAC quando conduzido com 50 sementes embebidas em $50 \mathrm{ml}$ de água destilada, enquanto para o cultivar Kada, o teste foi mais eficiente quando conduzido com 25 sementes embebidas em $50 \mathrm{ml}$ de água destilada. Entretanto, Sá (1999) observou para cultivares de tomate Petomech e Santa Clara que a variação no número de sementes usadas $(25,50$ e 100 sementes) não afeta os valores de condutividade elétrica.

Além da variação do número de sementes da amostra, o tempo de embebição das sementes tem efeito decisivo na capacidade do teste permitir distinguir diferenças de qualidade de lotes (Vieira e Krzyzanowski, 1999). Tradicionalmente, o teste de condutividade elétrica é conduzido utilizando-se o período de embebição de 24 horas; contudo, recentemente vários trabalhos indicaram a possibilidade de utilização de um período mais curto de embebição das sementes. Oliveira e Novembre (2005), avaliando a condutividade elétrica de sementes de pimentão, observaram que a embebição das sementes por apenas duas horas já é eficiente para a avaliação do potencial fisiológico. Em quiabo, Dias et al. (1998) verificaram que, após o período de quatro horas de condicionamento, foi possível a identificação de lotes de melhor desempenho. Dutra e Vieira (2006) consideraram que o período de 8 horas de embebição foi adequado para distinguir lotes de alto e baixo vigor de sementes de abobrinha. Já para sementes de cebola, Dias et al. (2006) concluíram que para sementes embebidas por 24 horas houve maior estratificação dos lotes em níveis de vigor. Rodo et al. (1998) realizaram um experimento utilizando dois cultivares de sementes de tomate e observaram que, independentemente do cultivar utilizado, a partir de quatro horas de embebição o teste de condutividade elétrica já permitiu a separação dos lotes.

Assim, o trabalho teve o objetivo de estudar, em sementes de pimenta, os efeitos do número de sementes, quantidade de água utilizada e o período de embebição nos valores de condutividade elétrica e verificar a eficiência deste teste para a avaliação do vigor das sementes.

\section{MATERIAL E MÉTODOS}

O experimento foi desenvolvido no Laboratório de Análises de Sementes do Departamento de Fitotecnia, Universidade Federal de Viçosa - MG, utilizando-se cinco lotes de sementes de pimenta, variedade Amarela Comprida. Foram realizados os seguintes testes e determinações: Grau de umidade - realizado pelo método da estufa a $105 \pm 3^{\circ} \mathrm{C}$, utilizando-se duas subamostras para cada lote, sendo os resultados expressos em porcentagem (Brasil, 1992). Germinação - conduzido com quatro subamostras de 50 sementes distribuídas sobre três folhas de papel germitest umedecidas com $6 \mathrm{~mL}$ de $\mathrm{KNO}_{3}$, em caixas plásticas $(11,0 \times 11,0 \times 3,5 \mathrm{~cm})$ e colocadas em germinador a $25^{\circ} \mathrm{C}$. As avaliações foram feitas no décimo e décimo sétimo dia após a semeadura e os resultados expressos em porcentagem de plântulas normais. Primeira contagem de germinação - consistiu do registro da porcentagem de plântulas normais obtidas no décimo dia após a montagem do teste. Envelhecimento acelerado - inicialmente, 300 sementes foram distribuídas uniformemente sobre tela acoplada à caixa gerbox com $40 \mathrm{~mL}$ de água destilada. As caixas foram tampadas e mantidas em câmara BOD, a $42^{\circ} \mathrm{C}$, 
por 96h (Bhering et al., 2006). Decorrido este período, duas repetições de 50 sementes foram utilizadas para determinação do grau de umidade das sementes, pelo método da estufa a $105 \pm 3^{\circ} \mathrm{C}$ por $24 \mathrm{~h}$ e 4 repetições de 50 sementes foram submetidas ao teste de germinação, conforme descrito acima. Os resultados foram expressos em porcentagem de plântulas normais obtidas no décimo quarto dia após a semeadura. Comprimento de plântula - foram utilizadas quatro subamostras de 20 sementes, que foram semeadas em duas linhas traçadas ao longo da extremidade superior do papel germitest, colocado na base de caixas plásticas (tipo gerbox), usando-se a tampa encaixada sobre a base da caixa para fixar as sementes. Foram utilizadas duas folhas de papel germitest umedecidas com volume de $\mathrm{KNO}_{3}$ equivalente a 2,5 vezes o peso do papel seco. As caixas plásticas foram colocadas em germinador a $25^{\circ} \mathrm{C}$, distribuídas inclinadamente nas bandejas formando um ângulo de $45^{\circ}$. A avaliação foi realizada aos 10 dias após a semeadura, tomando-se o comprimento, em milímetros, das plântulas normais. Condutividade elétrica - foram estudadas variações no número de sementes ( 25 e 50 sementes $)$, volume de água destilada $(25$ e $50 \mathrm{~mL})$ e período de embebição (1, 2, 3, 4, 5, 6, 7, 8, 12, 16, 24 e 48 horas). Foram avaliadas quatro repetições de 50 sementes, fisicamente puras, pesadas com precisão de três casas decimais $(0,001 \mathrm{~g})$, colocadas para embeber em água e mantidas em germinador a $25^{\circ} \mathrm{C}$. Após cada período de embebição, a condutividade elétrica da solução foi determinada por meio de leituras em condutivímetro DIGIMED, modelo 31, com os resultados expressos em $\mu \mathrm{S} . \mathrm{cm}^{-1} \cdot \mathrm{g}^{-1}$ de semente. Procedimento estatístico - o delineamento experimental utilizado foi o inteiramente casualizado, com quatro repetições. Os dados obtidos nos testes de condutividade elétrica foram analisados em esquema fatorial $5 \times 2 \times 2 \times 12$ (5 lotes, 2 números de semente, 2 volumes de água e 12 períodos de embebição) realizando-se a comparação entre as médias pelo teste de Tukey, a 5\% de probabilidade.

\section{RESULTADOS E DISCUSSÃO}

De acordo com a Tabela 1, verifica-se que houve uniformidade no teor de água das sementes, uma vez que a variação entre os lotes foi de até 1,46 pontos percentuais, o que é inferior à amplitude máxima aceita que é de $1 \mathrm{a} 2$ pontos percentuais, ficando evidente a consistência dos resultados, além deste fator não ter influenciado os testes empregados (Loeffler et al, 1988, Marcos Filho, 1999).

Ainda na Tabela 1, não foram observadas diferenças de qualidade entre os cinco lotes de sementes de pimenta pelos testes de germinação e envelhecimento acelerado, sendo os valores em porcentagens estatisticamente iguais. Entretanto, na primeira contagem de germinação, observouse melhor desempenho para o lote 4 , apesar de não ter diferido estatisticamente dos lotes 3 e 5 , enquanto os lotes 1 e 2 mostraram-se menos vigorosos. O lote 1 também foi significativamente inferior aos demais lotes pelo teste de comprimento de plântula, enquanto o lote 4 apresentou-se com maior vigor. Assim, de acordo com a caracterização dos lotes de sementes de pimenta, constatou-se que na maioria dos testes empregados houve semelhança quanto ao alto vigor do lote 4 e menor vigor do lote 1 . Cabe ressaltar que, os testes de germinação e envelhecimento acelerado não foram capazes de ordenar os lotes quanto ao nível de vigor, já os testes de primeira contagem de germinação e comprimento de plântula mostraram-se mais sensíveis às diferenças de qualidade entre os lotes, estratificando-os em três níveis de potencial fisiológico, ou seja, alto vigor para o lote 4, vigor intermediário para os lotes 3 e 5 e menor vigor para o lote. Observa-se que pelos resultados de comprimento de

TABELA 1. Caracterização da qualidade de cinco lotes de sementes de pimenta pelo teor de água (TA) e testes de germinação (TG), primeira contagem (PC), envelhecimento acelerado (EA) e comprimento de plântula (CP).

\begin{tabular}{cccccc}
\hline Lotes & TA (\%) & TG (\%) & PC (\%) & EA (\%) & CP (mm) \\
\hline 1 & 10,17 & $94 \mathrm{~A}$ & $63 \mathrm{~B}$ & $90 \mathrm{~A}$ & $47,2 \mathrm{~B}$ \\
2 & 10,85 & $89 \mathrm{~A}$ & $60 \mathrm{~B}$ & $91 \mathrm{~A}$ & $51,9 \mathrm{AB}$ \\
3 & 9,39 & $89 \mathrm{~A}$ & $72 \mathrm{AB}$ & $97 \mathrm{~A}$ & $57,2 \mathrm{AB}$ \\
4 & 9,96 & $89 \mathrm{~A}$ & $79 \mathrm{~A}$ & $91 \mathrm{~A}$ & $60,2 \mathrm{~A}$ \\
5 & 9,53 & $90 \mathrm{~A}$ & $74 \mathrm{AB}$ & $97 \mathrm{~A}$ & $59,9 \mathrm{AB}$ \\
\hline CV $(\%)$ & - & 6,89 & 9,65 & 3,94 & 10,25 \\
\hline
\end{tabular}

Na coluna, médias seguidas da mesma letra maiúscula não diferem entre si, pelo teste de Tukey, a $5 \%$ de probabilidade. 
plântulas, as sementes do lote 4 apresentaram vigor superior em relação aos demais lotes. Considerou-se as sementes dos lotes 2, 3 e 5 de vigor intermediário e as do lote 1 como de baixo vigor.

Pelas Tabelas 2 e 3, observou-se que o teste de condutividade elétrica utilizando-se 25 sementes, independentemente da quantidade de água (25 e $50 \mathrm{~mL}$ ), não foi eficiente na separação dos lotes em diferentes níveis de qualidade fisiológica para sementes de pimenta; no entanto, pesquisa realizada com sementes de pimentão por Oliveira e Novembre (2005), utilizando 25 sementes, conseguiram eficiência para a ordenação de lotes quanto à qualidade fisiológica.

Em todas as combinações do teste de condutividade elétrica (número de sementes e volume de água) podese verificar que os eletrólitos lixiviados pelas sementes aumentaram durante a embebição, constatação feita também por outros autores (Rodo et al., 1998; Dias et al., 2006; RoveriJosé et al., 2001). Contudo, a liberação foi consideravelmente maior quando as sementes, independente do número, foram embebidas em $25 \mathrm{~mL}$ de água destilada, fato este devido à maior concentração da solução ao reduzir o conteúdo de água. Resultados semelhantes foram encontrados por Dutra e Vieira (2006).

Analisando a Tabela 4, verificou-se que após uma hora de embebição, os lotes 4 e 5 destacaram-se dos demais, apresentando valores de condutividade sensivelmente inferiores aos dos outros lotes. Estes resultados corroboram com os resultados obtidos pelos testes utilizados para avaliação da qualidade das sementes (tabela 1). Oliveira e Novembre (2005), trabalhando com sementes de pimentão, também conseguiram identificar o lote de maior vigor com apenas uma hora de imersão das sementes. Também houve concordância quanto ao desempenho inferior do lote 2 devido ao maior conteúdo de exsudados lixiviados pelas sementes, mostrando maior grau de deterioração de suas membranas. É oportuno mencionar que também com sete horas de embebição confirmou-se esta classificação dos lotes quanto ao nível de vigor das sementes de pimenta.

Vale ressaltar a similaridade de ordenação dos lotes tanto no período de uma hora como de sete horas de embebição, indicando que a condutividade manteve a mesma tendência durante estes períodos, permitindo a possibilidade de realização do teste em menor tempo e, conseqüentemente a obtenção mais rápida dos

TABELA 2. Resultados do teste de condutividade elétrica - embebição de 25 sementes em 25 mL de água destilada, por períodos de $1,2,3,4,5,6,7,8,12,16,24$ e 48 horas de cinco lotes de sementes de pimenta.

\begin{tabular}{|c|c|c|c|c|c|c|}
\hline \multirow{3}{*}{ Lotes } & \multicolumn{6}{|c|}{ Condutividade elétrica $\left(\mu \mathrm{S} . \mathrm{cm}^{-1} \cdot \mathrm{g}^{-1}\right.$ semente $)$} \\
\hline & \multicolumn{6}{|c|}{ Período de embebição (hora) } \\
\hline & 1 & 2 & 3 & 4 & 5 & 6 \\
\hline 1 & $417,73 \mathrm{~A}$ & $462,03 \mathrm{~A}$ & $474,91 \mathrm{~A}$ & $476,02 \mathrm{~A}$ & $483,62 \mathrm{~A}$ & $484,83 \mathrm{~A}$ \\
\hline 2 & $421,69 \mathrm{~A}$ & $456,00 \mathrm{~A}$ & $468,86 \mathrm{~A}$ & $471,44 \mathrm{~A}$ & $481,42 \mathrm{~A}$ & $490,17 \mathrm{~A}$ \\
\hline 3 & $374,33 \mathrm{~A}$ & $451,92 \mathrm{~A}$ & $429,53 \mathrm{~A}$ & $432,74 \mathrm{~A}$ & $441,64 \mathrm{~A}$ & $449,99 \mathrm{~A}$ \\
\hline 4 & $390,04 \mathrm{~A}$ & $429,81 \mathrm{~A}$ & $448,09 \mathrm{~A}$ & $450,17 \mathrm{~A}$ & $462,87 \mathrm{~A}$ & $462,91 \mathrm{~A}$ \\
\hline 5 & $372,48 \mathrm{~A}$ & $404,75 \mathrm{~A}$ & $417,88 \mathrm{~A}$ & $421,62 \mathrm{~A}$ & $428,99 \mathrm{~A}$ & $432,72 \mathrm{~A}$ \\
\hline \multirow[t]{2}{*}{$\mathrm{CV}(\%)$} & 12,09 & 12,63 & 11,94 & 11,74 & 11,62 & 11,63 \\
\hline & \multicolumn{6}{|c|}{ Condutividade elétrica $\left(\mu \mathrm{S} . \mathrm{cm}^{-1} \cdot \mathrm{g}^{-1}\right.$ semente $)$} \\
\hline \multirow[t]{2}{*}{ Lotes } & \multicolumn{6}{|c|}{ Período de embebição (hora) } \\
\hline & 7 & 8 & 12 & 16 & 24 & 48 \\
\hline 1 & $489,98 \mathrm{~A}$ & $491,74 \mathrm{~A}$ & $498,88 \mathrm{~A}$ & $498,88 \mathrm{~A}$ & $499,34 \mathrm{~A}$ & $499,34 \mathrm{~A}$ \\
\hline 2 & $496,24 \mathrm{~A}$ & $498,49 \mathrm{~A}$ & $517,08 \mathrm{~A}$ & $517,08 \mathrm{~A}$ & $519,74 \mathrm{~A}$ & $521,33 \mathrm{~A}$ \\
\hline 3 & $455,21 \mathrm{~A}$ & $458,85 \mathrm{~A}$ & $465,47 \mathrm{~A}$ & $471,06 \mathrm{~A}$ & $480,62 \mathrm{~A}$ & $482,32 \mathrm{~A}$ \\
\hline 4 & $467,10 \mathrm{~A}$ & $472,86 \mathrm{~A}$ & $479,65 \mathrm{~A}$ & $480,62 \mathrm{~A}$ & $489,35 \mathrm{~A}$ & $489,65 \mathrm{~A}$ \\
\hline 5 & $435,75 \mathrm{~A}$ & $441,38 \mathrm{~A}$ & $451,39 \mathrm{~A}$ & $453,37 \mathrm{~A}$ & $459,69 \mathrm{~A}$ & $460,12 \mathrm{~A}$ \\
\hline CV (\%) & 11,75 & 11,70 & 11,41 & 11,16 & 10,69 & 10,72 \\
\hline
\end{tabular}

Na coluna, médias seguidas da mesma letra maiúscula não diferem entre si, pelo teste de Tukey, a 5\% de probabilidade. 
TABELA 3. Resultados do teste de condutividade elétrica - embebição de 25 sementes em 50 mL de água destilada, por períodos de $1,2,3,4,5,6,7,8,12,16,24$ e 48 horas de cinco lotes de sementes de pimenta.

\begin{tabular}{|c|c|c|c|c|c|c|}
\hline \multirow{3}{*}{ Lotes } & \multicolumn{6}{|c|}{ Condutividade elétrica $\left(\mu \mathrm{S} . \mathrm{cm}^{-1} \cdot \mathrm{g}^{-1}\right.$ semente $)$} \\
\hline & \multicolumn{6}{|c|}{ Período de embebição (hora) } \\
\hline & 1 & 2 & 3 & 4 & 5 & 6 \\
\hline 1 & $218,04 \mathrm{~A}$ & $243,06 \mathrm{~A}$ & $249,28 \mathrm{~A}$ & $252,05 \mathrm{~A}$ & $255,69 \mathrm{~A}$ & $256,91 \mathrm{~A}$ \\
\hline 2 & $220,16 \mathrm{~A}$ & $244,00 \mathrm{~A}$ & $252,28 \mathrm{~A}$ & $254,45 \mathrm{~A}$ & $258,11 \mathrm{~A}$ & $262,54 \mathrm{~A}$ \\
\hline 3 & $210,56 \mathrm{~A}$ & $234,07 \mathrm{~A}$ & $238,47 \mathrm{~A}$ & $240,35 \mathrm{~A}$ & $245,20 \mathrm{~A}$ & 246,19 A \\
\hline 4 & $218,47 \mathrm{~A}$ & $237,97 \mathrm{~A}$ & $246,61 \mathrm{~A}$ & $249,37 \mathrm{~A}$ & $254,93 \mathrm{~A}$ & $259,24 \mathrm{~A}$ \\
\hline 5 & $223,20 \mathrm{~A}$ & $240,09 \mathrm{~A}$ & $247,06 \mathrm{~A}$ & $248,81 \mathrm{~A}$ & $252,94 \mathrm{~A}$ & $256,00 \mathrm{~A}$ \\
\hline \multirow[t]{2}{*}{$\mathrm{CV}(\%)$} & 9,23 & 9,27 & 9,14 & 9,07 & 8,80 & 8,89 \\
\hline & \multicolumn{6}{|c|}{ Condutividade elétrica $\left(\mu \mathrm{S} . \mathrm{cm}^{-1} \cdot \mathrm{g}^{-1}\right.$ semente $)$} \\
\hline \multirow[t]{2}{*}{ Lotes } & \multicolumn{6}{|c|}{ Período de embebição (hora) } \\
\hline & 7 & 8 & 12 & 16 & 24 & 48 \\
\hline 1 & $260,49 \mathrm{~A}$ & $263,21 \mathrm{~A}$ & $267,50 \mathrm{~A}$ & $272,17 \mathrm{~A}$ & $275,21 \mathrm{~A}$ & $277,32 \mathrm{~A}$ \\
\hline 2 & $264,22 \mathrm{~A}$ & $268,37 \mathrm{~A}$ & $271,13 \mathrm{~A}$ & $274,95 \mathrm{~A}$ & $276,94 \mathrm{~A}$ & $281,36 \mathrm{~A}$ \\
\hline 3 & $247,61 \mathrm{~A}$ & $252,06 \mathrm{~A}$ & $255,88 \mathrm{~A}$ & $262,03 \mathrm{~A}$ & $264,03 \mathrm{~A}$ & $266,71 \mathrm{~A}$ \\
\hline 4 & $261,88 \mathrm{~A}$ & $265,08 \mathrm{~A}$ & $268,28 \mathrm{~A}$ & $272,18 \mathrm{~A}$ & $278,99 \mathrm{~A}$ & $280,52 \mathrm{~A}$ \\
\hline 5 & $257,87 \mathrm{~A}$ & $259,56 \mathrm{~A}$ & $271,58 \mathrm{~A}$ & $271,58 \mathrm{~A}$ & $275,41 \mathrm{~A}$ & $279,16 \mathrm{~A}$ \\
\hline CV (\%) & 8,87 & 8,88 & 8,50 & 8,47 & 8,30 & 8,19 \\
\hline
\end{tabular}

Na coluna, médias seguidas da mesma letra maiúscula não diferem entre si, pelo teste de Tukey, a $5 \%$ de probabilidade.

TABELA 4. Resultados do teste de condutividade elétrica - embebição de 50 sementes em 25 mL de água destilada, por períodos de $1,2,3,4,5,6,7,8,12,16,24$ e 48 horas de cinco lotes de sementes de pimenta.

\begin{tabular}{|c|c|c|c|c|c|c|}
\hline \multirow{3}{*}{ Lotes } & \multicolumn{6}{|c|}{ Condutividade elétrica $\left(\mu \mathrm{S} . \mathrm{cm}^{-1} \cdot \mathrm{g}^{-1}\right.$ semente $)$} \\
\hline & \multicolumn{6}{|c|}{ Período de embebição (hora) } \\
\hline & 1 & 2 & 3 & 4 & 5 & 6 \\
\hline 1 & $389,96 \mathrm{BC}$ & $418,45 \mathrm{~B}$ & $428,53 \mathrm{AB}$ & $431,70 \mathrm{AB}$ & $437,13 \mathrm{AB}$ & $441,19 \mathrm{AB}$ \\
\hline 2 & $394,86 \mathrm{C}$ & $419,17 \mathrm{~B}$ & $435,46 \mathrm{~B}$ & $437,97 \mathrm{~B}$ & $444,76 \mathrm{~B}$ & $452,48 \mathrm{~B}$ \\
\hline 3 & $339,83 \mathrm{AB}$ & $357,51 \mathrm{~A}$ & $378,37 \mathrm{AB}$ & $383,62 \mathrm{AB}$ & $387,03 \mathrm{AB}$ & $390,00 \mathrm{AB}$ \\
\hline 4 & $331,81 \mathrm{~A}$ & $360,83 \mathrm{AB}$ & $379,22 \mathrm{AB}$ & $380,30 \mathrm{AB}$ & $384,48 \mathrm{AB}$ & $387,69 \mathrm{AB}$ \\
\hline 5 & $333,16 \mathrm{~A}$ & $354,05 \mathrm{~A}$ & $367,49 \mathrm{~A}$ & $369,76 \mathrm{~A}$ & $374,44 \mathrm{~A}$ & $376,06 \mathrm{~A}$ \\
\hline \multirow[t]{2}{*}{$\mathrm{CV}(\%)$} & 6,55 & 6,99 & 7,42 & 7,41 & 7,18 & 7,23 \\
\hline & \multicolumn{6}{|c|}{ Condutividade elétrica $\left(\mu \mathrm{S} . \mathrm{cm}^{-1} \cdot \mathrm{g}^{-1}\right.$ semente $)$} \\
\hline \multirow[t]{2}{*}{ Lotes } & \multicolumn{6}{|c|}{ Período de embebição (hora) } \\
\hline & 7 & 8 & 12 & 16 & 24 & 48 \\
\hline 1 & $446,63 \mathrm{BC}$ & $452,02 \mathrm{~B}$ & $454,19 \mathrm{~B}$ & $454,64 \mathrm{~B}$ & $455,82 \mathrm{AB}$ & $457,20 \mathrm{AB}$ \\
\hline 2 & $456,22 \mathrm{C}$ & $457,76 \mathrm{~B}$ & $458,81 \mathrm{~B}$ & $459,63 \mathrm{~B}$ & $463,49 \mathrm{~B}$ & $466,25 \mathrm{~B}$ \\
\hline 3 & $392,72 \mathrm{ABC}$ & $398,15 \mathrm{AB}$ & $401,43 \mathrm{AB}$ & $404,36 \mathrm{AB}$ & $409,31 \mathrm{AB}$ & $409,31 \mathrm{AB}$ \\
\hline 4 & $389,35 \mathrm{AB}$ & $393,25 \mathrm{AB}$ & $394,89 \mathrm{AB}$ & $395,91 \mathrm{AB}$ & $398,27 \mathrm{AB}$ & $398,88 \mathrm{AB}$ \\
\hline 5 & $378,40 \mathrm{~A}$ & $381,02 \mathrm{~A}$ & $381,92 \mathrm{~A}$ & $382,60 \mathrm{~A}$ & $389,60 \mathrm{~A}$ & $389,96 \mathrm{~A}$ \\
\hline $\mathrm{CV}(\%)$ & 7,05 & 7,21 & 7,24 & 7,48 & 6,95 & 7,05 \\
\hline
\end{tabular}

Na coluna, médias seguidas da mesma letra maiúscula não diferem entre si, pelo teste de Tukey, a $5 \%$ de probabilidade. 
TABELA 5. Resultados do teste de condutividade elétrica - embebição de 50 sementes em 50 mL de água destilada, por períodos de $1,2,3,4,5,6,7,8,12,16,24$ e 48 horas de cinco lotes de sementes de pimenta.

\begin{tabular}{|c|c|c|c|c|c|c|}
\hline \multirow{3}{*}{ Lotes } & \multicolumn{6}{|c|}{ Condutividade elétrica $\left(\mu \mathrm{S} . \mathrm{cm}^{-1} \cdot \mathrm{g}^{-1}\right.$ semente $)$} \\
\hline & \multicolumn{6}{|c|}{ Período de embebição (hora) } \\
\hline & 1 & 2 & 3 & 4 & 5 & 6 \\
\hline 1 & $213,04 \mathrm{~B}$ & $231,80 \mathrm{~B}$ & $239,58 \mathrm{~B}$ & $241,80 \mathrm{~B}$ & $242,57 \mathrm{~B}$ & $243,73 \mathrm{~B}$ \\
\hline 2 & $199,52 \mathrm{AB}$ & $215,13 \mathrm{AB}$ & $221,87 \mathrm{AB}$ & $223,47 \mathrm{AB}$ & $225,21 \mathrm{AB}$ & $226,20 \mathrm{AB}$ \\
\hline 3 & $185,86 \mathrm{~A}$ & $204,84 \mathrm{~A}$ & $211,47 \mathrm{~A}$ & $213,04 \mathrm{~A}$ & $214,75 \mathrm{~A}$ & $216,39 \mathrm{~A}$ \\
\hline 4 & $195,38 \mathrm{AB}$ & $207,47 \mathrm{~A}$ & $214,48 \mathrm{AB}$ & $216,75 \mathrm{AB}$ & $218,87 \mathrm{AB}$ & $220,67 \mathrm{AB}$ \\
\hline 5 & $194,44 \mathrm{AB}$ & $208,57 \mathrm{AB}$ & $215,16 \mathrm{AB}$ & $217,30 \mathrm{AB}$ & $218,28 \mathrm{AB}$ & $220,43 \mathrm{AB}$ \\
\hline CV $(\%)$ & 5,43 & 5,05 & 5,25 & 5,39 & 5,20 & 5,11 \\
\hline \multirow{3}{*}{ Lotes } & \multicolumn{6}{|c|}{ Condutividade elétrica $\left(\mu \mathrm{S} . \mathrm{cm}^{-1} \cdot \mathrm{g}^{-1}\right.$ semente $)$} \\
\hline & \multicolumn{6}{|c|}{ Período de embebição (hora) } \\
\hline & 7 & 8 & 12 & 16 & 24 & 48 \\
\hline 1 & $245,44 \mathrm{~A}$ & $247,65 \mathrm{~A}$ & $249,60 \mathrm{~A}$ & $252,45 \mathrm{~A}$ & $254,83 \mathrm{~A}$ & $254,98 \mathrm{~A}$ \\
\hline 2 & $228,58 \mathrm{~A}$ & $230,33 \mathrm{~A}$ & $233,24 \mathrm{~A}$ & $237,08 \mathrm{~A}$ & $241,05 \mathrm{~A}$ & $242,20 \mathrm{~A}$ \\
\hline 3 & $218,96 \mathrm{~A}$ & $220,96 \mathrm{~A}$ & $223,60 \mathrm{~A}$ & $227,66 \mathrm{~A}$ & $235,08 \mathrm{~A}$ & $235,08 \mathrm{~A}$ \\
\hline 4 & $222,23 \mathrm{~A}$ & $224,30 \mathrm{~A}$ & $227,81 \mathrm{~A}$ & $231,13 \mathrm{~A}$ & $235,52 \mathrm{~A}$ & $236,36 \mathrm{~A}$ \\
\hline 5 & $222,30 \mathrm{~A}$ & $223,28 \mathrm{~A}$ & $225,12 \mathrm{~A}$ & $229,27 \mathrm{~A}$ & $230,59 \mathrm{~A}$ & $230,59 \mathrm{~A}$ \\
\hline CV $(\%)$ & 5,48 & 5,25 & 5,21 & 5,18 & 5,32 & 5,39 \\
\hline
\end{tabular}

Na coluna, médias seguidas da mesma letra maiúscula não diferem entre si, pelo teste de Tukey, a 5\% de probabilidade.

resultados. Resultados similares foram verificados em pesquisa realizada por Dutra e Vieira (2006) com sementes de abobrinha, onde a classificação de lote similar foi obtida para oito e 24 horas de embebição. Outros autores também reduziram o tempo de embebição em relação às 24 horas indicadas (Rodo et al., 1998; Dias et al., 1998; Oliveira e Novembre, 2005).

Pela Tabela 5, verifica-se que o lote 3 foi o de maior vigor e o de menor vigor, o lote 1 , entre uma a seis horas de embebição. Após este período, de sete a 48 horas, o teste de condutividade elétrica não foi capaz de estratificar os lotes quanto aos níveis de vigor, o que pode ser explicado pelo vazamento de solutos das diferentes células por difusão passiva durante os estádios inicias de hidratação das membranas, que pode ocorrer mais rápida ou lentamente dependendo do sistema de reorganização da dupla camada lipídica.

Verifica-se, no presente trabalho, que o teste de condutividade elétrica foi eficiente para avaliação do potencial fisiológico dos lotes de pimenta, utilizando-se 50 sementes embebidas em $25 \mathrm{~mL}$ de água destilada, nas avaliações feitas com uma hora de embebição.

\section{CONCLUSÃO}

No teste de condutividade elétrica em sementes de pimenta, maior estratificação dos lotes em níveis de vigor foi obtida quando foram utilizadas amostras de 50 sementes, embebidas em $25 \mathrm{~mL}$ de água destilada, avaliando-se a condutividade elétrica após uma hora de embebição.

\section{REFERÊNCIAS}

ASSOCIATION OF OFFICIAL SEED ANALYSTS. Seed vigor testing handbook. Lincoln, 2002. 105p. (Contribution, 32).

BHERING, M.C.; DIAS, D.C.F.S.; VIDIGAL， D.S.; NAVEIRA, D.S.P. Teste de envelhecimento acelerado em sementes de pimenta. Revista Brasileira de Sementes, Pelotas, v.28, n.3; p.64-71, 2006.

BRASIL, Ministério da Agricultura e Reforma Agrária. Regras para Análise de Sementes. Brasília, 1992. 365p.

DIAS, D.C.F.S.; MARCOS FILHO, J. Testes de condutividade elétrica para avaliação do vigor de sementes de soja ( Glycine 
$\max ($ L.) Merrill). Scientia Agrícola, Piracicaba, v.53, n.1, p.31-42, 1996.

DIAS, D.C.F.S.; VIEIRA, A.N.; BHERING, M.C. Condutividade elétrica e lixiviação de potássio para avaliação do vigor de sementes de hortaliças: feijão-de-vagem e quiabo. Revista Brasileira de Sementes, Brasília, v.20, n.2, p.408-413, 1998.

DUTRA, A.; VIEIRA, R.D. Teste de condutividade elétrica para a avaliação do vigor de sementes de abobrinha. Revista Brasileira de Sementes, Pelotas, v.28, n.2, p.117-122, 2006.

GASPAR, C.M.; NAKAGAWA, J. Teste de condutividade elétrica em função do número de sementes e da quantidade de água para sementes de milheto. Revista Brasileira de Sementes, Londrina, v.24, n.2, p.70-76, 2002.

LOEFFLER, T.M.; TEKRONY, D.M.; EGLI, B.D. The bulk conductivity test as on indicator of soybean seed quality. Journal of Seed Technology, Springfield, v.12, n.1, p.37-53, 1988.

MARCOS FILHO, J. Teste de envelhecimento acelerado. In: KRZYZANOWSKI, F.C.; VIEIRA, R.D.; FRANÇA NETO, J.B. (Eds.). Vigor de sementes: conceitos e testes. Londrina: ABRATES, p.3.1-3.24, 1999.

OLIVEIRA, S.R.S.; NOVEMBRE, A.D.L.C. Teste de condutividade elétrica para as sementes de pimentão. Revista Brasileira de Sementes, Pelotas, v.27, n.1, p.31-36, 2005.

RODO, A.B.; TILLMANN, M.A.A.; VILLELA, F.A.; SAMPAIO, N.V. Teste de condutividade elétrica em sementes de tomate. Revista Brasileira de Sementes, Brasília, v.20, n.1, p.29-38, 1998.

ROVERI-JOSÉ, S.C.B.; CARVALHO, M.L.M.; RODRIGUES, R. Teste de condutividade elétrica para avaliação da qualidade fisiológica de sementes de pimentão (Capsicum annuum 1.). Revista Brasileira de Sementes, Brasília, v. 23, n.1. p.55-61, 2001.

SÁ, M.E. Condutividade elétrica em sementes de tomate (Lycopersicon lycopersicum L.). Scientia Agricola, Piracicaba, v.56, n.1, p.13-19, 1999.

VANZOLINI, S.; NAKAGAWA, J. Teste de condutividade elétrica em genótipos de sementes de amendoim. Revista Brasileira de Sementes, Brasília, v.20, n.1, p.178-183, 1998.

VIEIRA, R.;KRZYZANOWSKI,F.C. Teste de condutividade elétrica. In: KRZYZANOWSKI, F.C.; VIEIRA, R.D.; FRANÇA NETO, J.B. (Eds.). Vigor de sementes: conceitos e testes. Londrina: ABRATES, p.4.1-4.26, 1999. 